\title{
Simulation Research on Ran-Assisted WLAN Offloading Scheme
}

\author{
Yuanying Shi, Guangyue Lu, Junmin Jiang ${ }^{1}$, Jiali Zhang \\ School of Telecommunication and Information Engineering, Xi'an University of Posts and Telecommunications, Xi'an, China \\ Email: shi_yuanying@163.com, tonylugy@163.com,jjunmin@yeah.net, zhangjiali_2011@163.com
}

How to cite this paper: Shi, Y.Y., Lu, G.Y., Jiang, J.M. and Zhang, J.L. (2017) Simulation Research on Ran-Assisted WLAN Offloading Scheme. Int. J. Communications, Network and System Sciences, 10, 324-332. https://doi.org/10.4236/ijcns.2017.105B032

Received: July 10, 2017

Accepted: August 1, 2017

Published: August 4, 2017

\begin{abstract}
Wireless Local Area Network (WLAN) offloading is an important approach to address the data traffic challenge faced by Long Term Evolution (LTE) network. Legacy offloading solutions based on the core network suffer from the limitations of load unbalance and user experience degradation. To solve this problem, 3GPP has recently proposed a radio access network (RAN) assisted WLAN offloading scheme. In this article, we thoroughly analyze the performance of this new scheme, with emphasis on the RAN auxiliary parameters and control rules defined in it. Furthermore, two kinds of offloading algorithms based on this scheme are proposed and compared with the traditional solutions by simulation. Results show that the RAN-assisted offloading scheme can increase average user throughput and leverage resources available in both LTE and WLAN systems.
\end{abstract}

\section{Keywords}

LTE, WLAN, RAN-Assisted, Traffic Offloading

\section{Introduction}

With the increasing popularity of modern terminals such as smart phones and tablets, the amount of mobile data traffic has recently been doubling every year. This unprecedented traffic demands have imposed significant challenge on Long Term Evolution (LTE) network, which forces mobile network operators to investigate ways to offload data traffic to different Radio Access Networks (RANs), such as Wireless Local Area Network (WLAN).

An effective offloading strategy plays an important role for efficiently use of the available resources in both LTE and WLAN networks as well as improvement of the end user's experience. A number of literatures such as [1] [2] [3] [4] [5] have discussed this topic and presented algorithms based on Access Network 
Discovery and Selection Function (ANDSF) [6]. However, these ANDSF-based solutions mainly focus on the core network (CN) and cannot adapt well to dynamic changes of radio environments (e.g. the access node load and channel quality), hence resulting in poor user experience or inadequate utilization of WLAN resources [7].

To realize effective balancing of user data traffic between LTE and WLAN networks, 3GPP has recently defined a new feature named "RAN-assisted WLAN interworking" in Release 12, which can provide the user equipment (UE) with information of radio signal strength and the load level of LTE cells and WLAN Access Points (APs). Several offloading algorithms based on this scheme have been proposed in [8] [9] [10]. However, to the authors' best knowledge, few works have studied it in a holistic view. In this paper, we will explore the RAN-assisted offloading scheme in detail and evaluate its performance by system simulation.

The remaining of the paper is organized as follows. Detailed description and analysis of the RAN-assisted WLAN offloading scheme is given in Section 2. Two kinds of RAN-assisted offloading algorithms for performance evaluation are proposed in Section 3. Simulation results and discussion are provided in Section 4 followed by conclusions in Section 5 .

\section{RAN-Assisted WLAN Offloading Scheme}

RAN-assisted WLAN offloading scheme is an enhancement to existing $\mathrm{CN}$ based solutions. In the latter case, the ANDSF only provides a UE with the identification of offloadable WLANs. Without knowledge of the WLAN load level and its quality of service, the UE can just adopt simple offloading policy, e.g. offloading when the indicated WLAN is available. Obviously, this kind of policy might result in poor user experience if the target WLAN is already overloaded. Hence, in RAN-assisted offloading, an eNodeB should collect more information about itself and neighboring WLAN networks, such as load condition, backhaul quality, offload preference, etc. An eNodeB might obtain WLAN information directly through an interface between them, or from the UE's reports. Since RAN-assisted offloading is a network assisted UE controlled solution, the eNodeB should send the UE the collected information as RAN-assisted parameters which will be used in offloading algorithms pre-configured in the UE. Table 1 lists the parameters defined in 3GPP TS 36.304 [11]. Deserved to be mentioned, an eNodeB doesn't need to use all of the parameters simultaneously, which means it can arbitrarily combine them. Accordingly, a UE should use the same set of parameters as its serving eNodeB.

For each parameter in Table 1, a pair of thresholds is defined. One is used for offloading from LTE to WLAN, and the other is from WLAN to LTE. An eNodeB periodically scans its cell load level and, if possible, acquires the states of the neighboring WLANs. When necessary, it will adjust the thresholds values and notify the UE directly or through an ANDSF server. The UE compares these thresholds with its physical layer measurements and information provided by 
the WLAN to decide whether to offload or not. For example, the UE will offload traffic to WLAN only if both the measured Reference Signal Received Power (RSRP) is below RSRPThreshold ${ }_{L T E->W L A N}$ and WLAN channel utilization is below ChannelUtilizationThreshold ${ }_{L T E->W L A N}$. RSRPThreshold LTE->WLAN $_{\text {is }}$ ise threshold of parameter RSRPmeas and ChannelUtilization Threshold ${ }_{\text {LTE- } \rightarrow W L A N}$ is that of ChannelUtilizationWLAN. The subscript means they are used for offloading from LTE to WLAN. Obviously, this policy provides an opportunity for the UE to make a more rational choice than just offloading traffic to WLAN when available.

There are two kinds of control granularity for RAN-assisted offloading scheme: coarse-grained and fine-grained. In the coarse-grained mechanism, an eNodeB sets the same thresholds for all target WLAN networks within a cell's coverage. It broadcasts the thresholds values in system information message to all UEs. In this case, the thresholds can't reflect differences between WLAN networks, e.g. their locations, so it can't balance the network load effectively. For the offloading scenario shown in Figure 1, the outermost circle represents a cell's actual coverage region and the dotted circle represents the range corre sponding to RSRPThreshold ${ }_{L T E-\triangle W L A N}$. It is clear that a UE will not offload traffic to WLAN \#1 even if it is in light load state because it doesn't meet the rule that the measured RSRP is below RSRPThreshold $d_{L T E->W L A N}$. Then WLAN \#1 will not be fully utilized unless the cell reduces the scope of the dotted circle by increas-

Table 1. RAN-assisted parameters defined in TS 36.104.

\begin{tabular}{cc}
\hline Parameter & Description \\
\hline $\begin{array}{c}\text { ChannelUtilizationWLAN } \\
\text { BackhaulRateDlWLAN } \\
\text { BackhaulRateUlWLAN } \\
\text { WLANRSS }\end{array}$ & $\begin{array}{r}\text { percentage of time that the AP senses the medium was busy } \\
\text { backhaul available downlink bandwidth of WLAN } \\
\text { backhaul available uplink bandwidth of WLAN }\end{array}$ \\
RSRPmeas & a measure of the radio frequency energy of received frames \\
RSRQmeas & the ratio of RSRPmeas to received signal strength for an LTE carrier \\
\hline
\end{tabular}

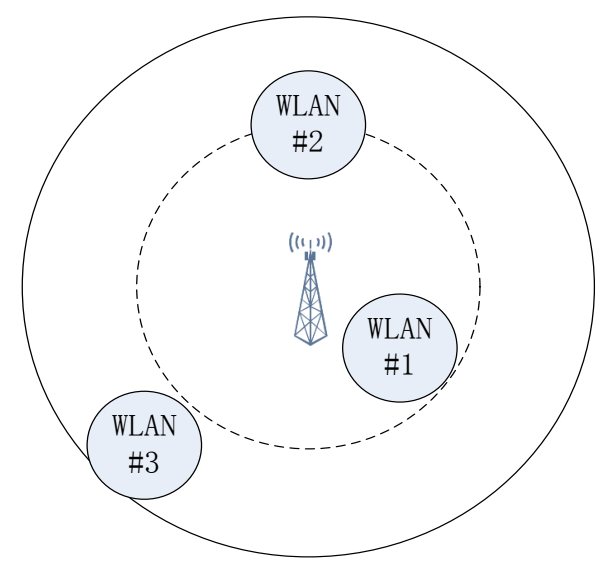

Figure 1. An example offloading scenario. 
ing RSRPThreshold ${ }_{L T E->W L A N}$. Hence, a fine-grained offloading control mechanism is required.

In the fine-grained mechanism, an eNodeB sets indiviual thresholds values for each UE. Thus it has a chance to take into account the UE's location and its surrounding radio environments. The thresholds must be sent to the specific UE by dedicated signaling. Therefore, this mechanism is only suitable for UE in LTE connected state. However, the thresholds values may keep valid for a period of time. During this period, the UE is allowed to use these values even if it has gone to idle state or has associated to a WLAN AP.

\section{Offloading Algorithms for Evaluation}

To evaluate the performance of the RAN-assisted offloading scheme, we present two specific algorithms with different control granularity. A simple algorithm based on the legacy offloading scheme is also described below for simulation comparison.

\subsection{Algorithm 1}

This algorithm represents the traditional $\mathrm{CN}$-based offloading solutions and is used as a reference for performance comparison. In this algorithm, a UE doesn't take into account the RAN-assisted information when it selects an offloading target. It means the UE will offload data traffic to WLAN networks whenever it finds the Received Signal Strength (RSS) of a WLAN AP is above the minimum threshold, which is $-96 \mathrm{dBm}$ in our simulation.

\subsection{Algorithm 2}

This algorithm represents the coarse-grained mechanism described in Section 2. It is based on the algorithm presented in [9] while employs a self-optimized strategy for thresholds adjustment.

In this algorithm, an eNodeB only monitors for changes in the LTE cell load and adjusts the RSRP thresholds accordingly. The LTE cell load is defined as the physical resource block utilization percentage in uplink or down-link direction. Two LTE cell load thresholds are pre-defined: LoadThresh $h_{L T E-h i g h}$ and LoadThresh ${ }_{L T E-l o w}$ If the LTE cell load is higher than LoadThresh $_{\text {LTE-high }}, R S R P$-Threshold LTE->WLAN $_{\text {will be }}$ increased to offload more data traffic to WLAN. If the LTE cell load is under

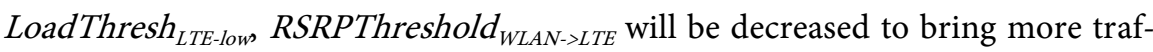
fic back to LTE.

A UE connected to LTE should check if the measured RSRP is below RSRPThreshold ${ }_{L T E->W L A N}$ and if it can find a WLAN AP with RSS higher than RSSThreshold ${ }_{L T E->W L A N}$. If both conditions are met, the UE will offload its traffic to WLAN. A UE associated with a WLAN AP will execute similar operation, i.e. if the RSRP is above RSRPThreshold ${ }_{\text {WLAN } \rightarrow \text { LTE }}$ and the RSS is lower than RSSThreshold $_{W L A N->L T E}$ it will bring the traffic back to LTE.

Main parameters for this algorithm and their simulation values are listed in Table 2. 
Table 2. Main parameters for Algorithm 2.

\begin{tabular}{|c|c|c|}
\hline Parameter & Description & Value \\
\hline LoadThresh $h_{\text {LTE-high }}$ & High LTE cell load threshold & $60 \%$ \\
\hline LoadThresh $_{\text {LTE-low }}$ & Low LTE cell load threshold & $40 \%$ \\
\hline$R S R P T h r_{L T E->W L A N-\text { initial }}$ & Initial value of $R S R P T h r e s h o l d_{L T E->W L A N}$ & $-109 \mathrm{dBm}$ \\
\hline$R S R P T h r_{W L A N->L T E-\text {-nitial }}$ & Initial value of $R S R P T h r e s h o l d W_{W L A N->L T E}$ & $-80 \mathrm{dBm}$ \\
\hline RSRP_Thr_Step & Step size of the RSRP threshold adjustment & $1 \mathrm{~dB}$ \\
\hline RSSThreshold $_{L T E->W L A N}$ & RSS threshold for offloading from LTE to WLAN & $-92 \mathrm{dBm}$ \\
\hline RSSThreshold $_{W L A N->L T E}$ & RSS threshold for offloading from WLAN to LTE & $-92 \mathrm{dBm}$ \\
\hline
\end{tabular}

\subsection{Algorithm 3}

This algorithm represents the fine-grained mechanism described in Section 2 and is an improvement of Algorithm 2. The algorithm takes into account both the LTE cell load and the WLAN load. The WLAN load is represented by the percentage of average channel busy time of a WLAN AP [10]. In addition to the LTE cell load thresholds described in Algorithm 2, two more load thresholds are defined for WLAN: LoadThesh AP-high $_{\text {and LoadThresh }}$ AP-low The RSRP and RSS thresholds are set and adjusted for each pair of LTE cell and WLAN AP. Table 3 describes the adjustment rules under various load conditions.

Then the eNodeB should send appropriate thresholds values to UEs based on their locations. Here we choose the values corresponding to the UE's serving cell and its nearest WLAN AP. The UE's operation is the same as that described in Algorithm 2.

Main parameters for this algorithm and their simulation values are captured in Table 4. Parameters already used in Algorithm 2 are not listed in the table.

\section{Simulation Scenario and Results}

To evaluate the proposed algorithms, we adopt a simulation scenario of an outdoor high traffic shopping center as shown in Figure 2. The deployment includes 7 LTE macro sites (red points in Figure 2) and 3 randomly distributed WLAN APs (blue points) for each LTE cell. Hence, the total number of LTE cells and WLAN APs are 21 and 63 respectively. The LTE network operates at $2 \mathrm{GHz}$ and uses a bandwidth of $10 \mathrm{MHz}$. The WLAN system modelling follows the IEEE $802.11 \mathrm{n}$ standard and operates at $2.4 \mathrm{GHz}$ with a bandwidth of $20 \mathrm{MHz}$. The transmission power of the eNodeB and the AP is $46 \mathrm{dBm}$ and $16 \mathrm{dBm}$ respectively.

50 UEs (orange points) are placed within each LTE cell coverage. Among them, 14 are randomly distributed, and 12 are concentrated around each AP. All UEs are static and can receive control messages from the eNodeB all the time.

User traffic is generated according to the non-full buffer ftp traffic model defined in 3GPP TR 36.814 [12]. The file size is 0.5 Mbytes and the time interval between end of download of previous file and the user request for the next file follows an exponential distribution with a mean of 4 seconds. 


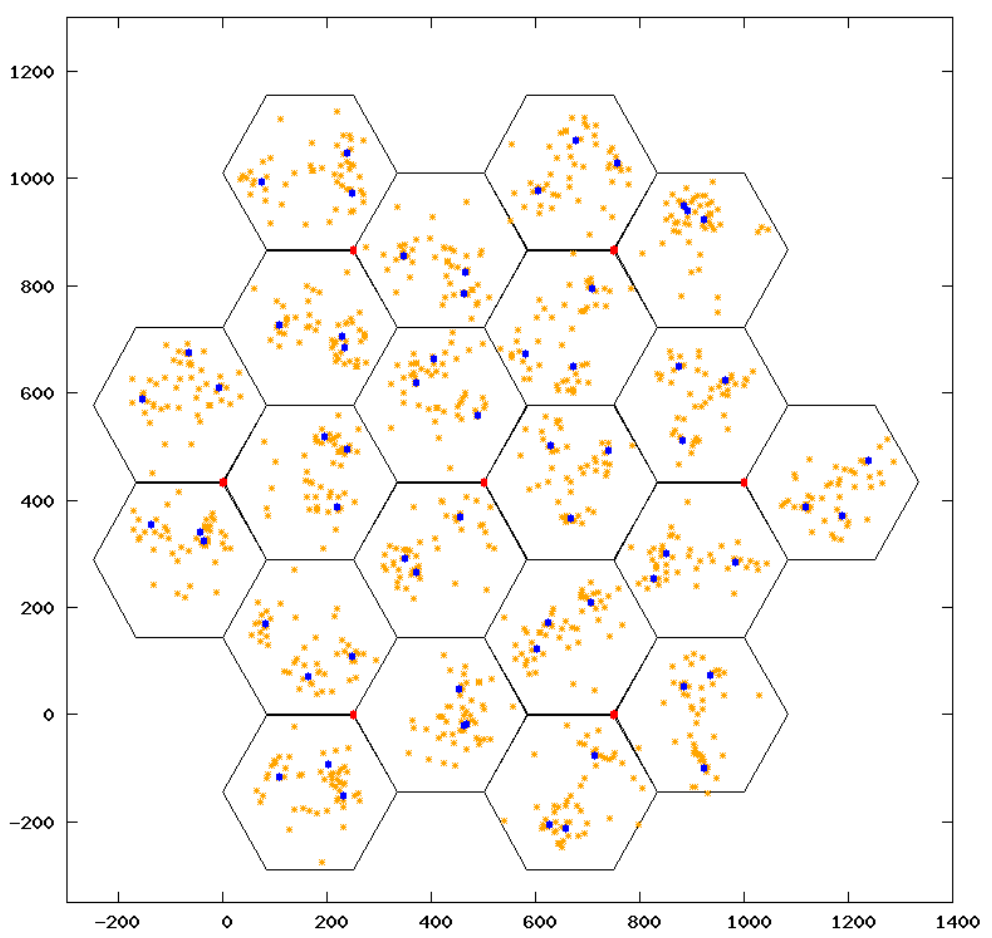

Figure 2. Simulation scenario.

Table 3. Adjustment rules for thresholds.

\begin{tabular}{|c|c|c|}
\hline LTE cell & WLAN AP & Adjustment rule \\
\hline overloaded & overloaded & no need to adjust \\
\hline overloaded & not overloaded & $\begin{array}{c}\text { increase } R S R P T h r e s h o l d \\
\text { decrease } \text { RSSThreshold }_{L T E->W L A N}\end{array}$ \\
\hline not overloaded & overloaded & $\begin{array}{l}\text { Increase } R S S T h r e s h o l d_{L T E->W L A N} \\
\text { decrease } R S R P T h r e s h o l d \\
\text { LTE->WLAN }\end{array}$ \\
\hline underloaded & not underloaded & $\begin{array}{c}\text { decrease RSRPThreshold } \\
\text { increase } R S S T h r e s h o l d \\
W L A N->L T E \\
\end{array}$ \\
\hline not underloaded & underloaded & $\begin{array}{l}\text { decrease } R S S T h r e s h o l d \\
\text { increase } R S R P T h r e s h o l d \\
\text { WLAN->LTE }\end{array}$ \\
\hline underloaded & underloaded & no need to adjust \\
\hline
\end{tabular}

Table 4. Main parameters for Algorithm 3.

\begin{tabular}{|c|c|c|}
\hline Parameter & Description & Value \\
\hline LoadThesh $_{A P-h i g h}$ & High WLAN AP load threshold & $60 \%$ \\
\hline LoadThresh $_{\text {AP-low }}$ & Low WLAN AP load threshold & $40 \%$ \\
\hline$R S S T h r_{L T E->W L A N-\text {-initial }}$ & Initial value of $R S S T h r e s h o l d d_{L T E->W L A N}$ & $-92 \mathrm{dBm}$ \\
\hline$R S S T h r_{W L A N->L T E-\text { initial }}$ & Initial value of RSSThreshold ${ }_{W L A N->L T E}$ & $-65 \mathrm{dBm}$ \\
\hline RSS_Thr_Step & Step size of the RSS threshold adjustment & $1 \mathrm{~dB}$ \\
\hline
\end{tabular}

Simulation results are shown in Figure 3 and Figure 4.

Figure 3 compares the average UE throughput in the downlink direction. It is obvious that Algorithm 1 has the minimum throughput because it offloads all data traffic to WLAN without considering its channel utilization level. There 


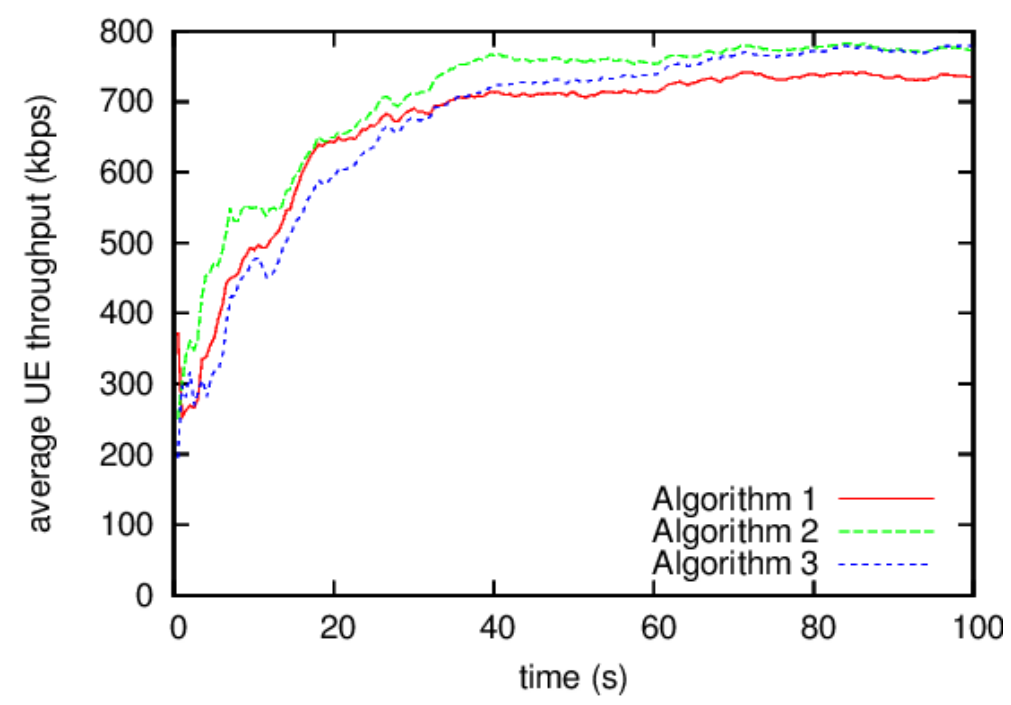

Figure 3. Comparison of average UE throughput.
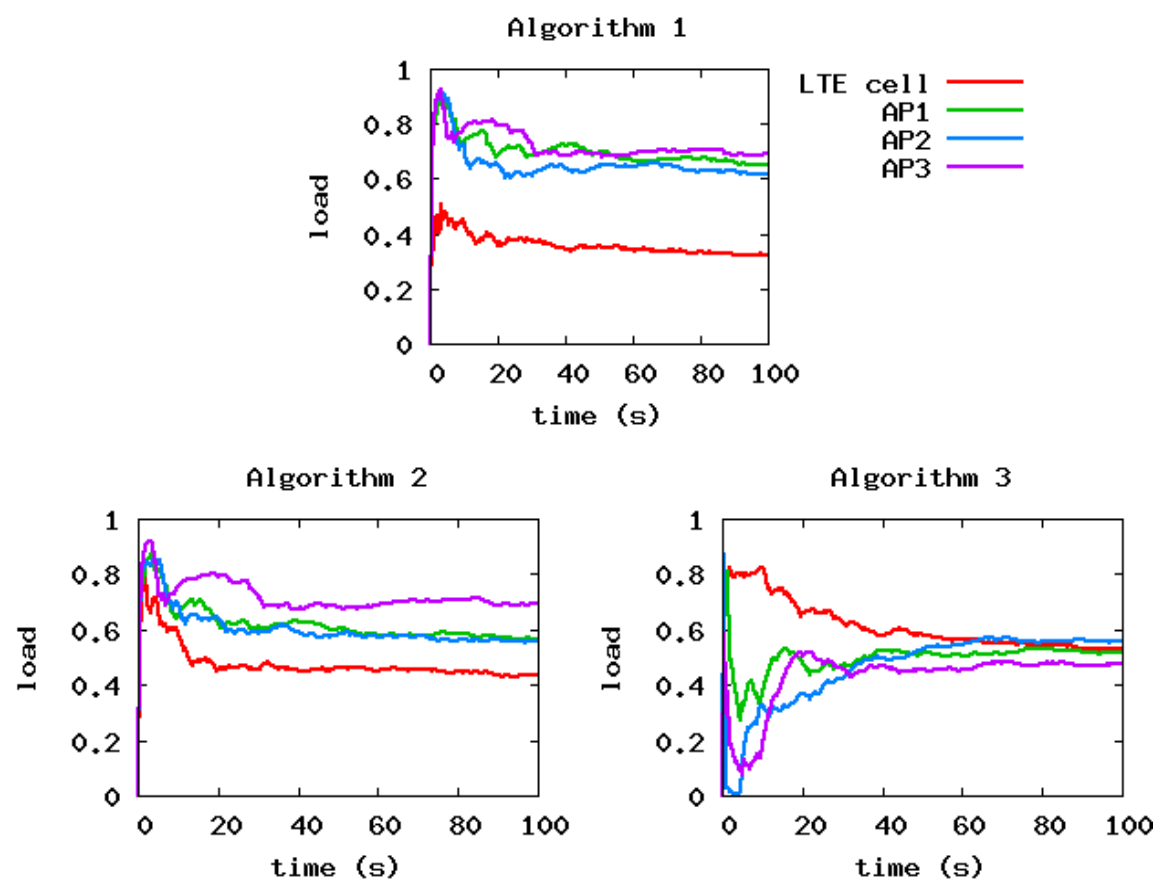

Figure 4. Load comparison of an example LTE cell and associated WLAN APs.

fore, the performance of the WLAN will decrease when it is in heavy load. At the same time, the resources in the LTE cell maybe have not been fully utilized.

Figure 3 also shows that at the beginning of the simulation, Algorithm 2 has a higher throughput than Algorithm 3. With the increase of time, the gap between them gets smaller and smaller. By the end of the simulation, the throughput of Algorithm 3 has exceeded a bit. It means that in the long term Algorithm 3 will have better performance, but it must spend more time to find out the optimized thresholds values.

Figure 4 compares the loads of an example LTE cell and all WLAN APs within its coverage. Apparently, in Algorithm 1, the user data traffic is not 
evenly distributed between the LTE cell and the WLANs because of the greedy use of WLAN resources. Algorithm 2 can improve this situation by instructing part of the UEs to offload their traffic from WLAN to the LTE cell. However, the effect is limited and there are obvious load differences between the WLAN APs. The reason is that the same thresholds values will have different effects on APs in different locations, as explained in Figure 1. In contrast, Algorithm 3 can achieve load balancing by setting optimized thresholds values for each UE.

Figure 4 also reflects the difference of convergence speed between Algorithm 2 and 3. Because both of them adopt self-optimized scheme, they need some time to find out the optimized thresholds configuration. Algorithm 2 takes about 30 seconds to stabilize, while Algorithm 3 needs another 30 seconds.

\section{Conclusion}

In this paper, we studied the RAN-assisted WLAN offloading scheme and evaluated its performance by system simulation. Results show that this scheme can improve average UE throughput and gain better load balancing than traditional CN-based solutions. Furthermore, a performance comparison is illustrated between the two kinds of control rules defined in RAN-assisted offloading and the simulation results reflect better property of the fine-grained mechanism.

We have not taken into account some control parameters such as WLAN backhaul rate in the proposed algorithms. Their impact on the system performance will be studied in our future work.

\section{Acknowledgements}

This research is supported by National Science and Technology Major Project of the Ministry of Science and Technology of China (2014ZX03001025).

\section{References}

[1] 3GPP TS 23.261 (2010) IP Flow Mobility and Seamless Wireless Local Area Network (WLAN) Offload.

[2] 3GPP TS 23.861 (2009) Network Based IP Flow Mobility.

[3] Dae Sun, K., Yujin, N., Yoshinori, K. and Hidetoshi, Y. (2013) Efficient ANDSFAssisted Wi-Fi Control for Mobile Data Offloading. Proc. Int. Conf. Wireless Communications and Mobile Computing, Cagliari, July 2013, 343-348.

[4] Nguyen, N. and Sato, T. (2014) A Proposal for Dynamic WLAN Selection for Mobile Data Offloading in Heterogeneous Network. Proc. IEEE Vehicular Technology Conference, Seoul, May 2014, 1-5.

[5] Chin-Yu, L., Fang-Yie, L., Jung-Chun, L., Castigiglione, A. and Palmieri, F. (2015) Heterogeneous Network Handover Using 3GPP ANDSF. Proc. Int. Conf. Advanced Information Networking and Applications, Gwangju, March 2015, 171-175.

[6] 3GPP TS 24.312 (2008) Access Network Discovery and Selection Function (ANDSF) Management Object (MO).

[7] 3GPP TR 37.834 (2014) Study on Wireless Local Area Network (WLAN)-3GPP Radio Interworking.

[8] István, Z.K., Daniela, L., Per-Henrik, M., Wang, Y., Relja, D. and Kathleen, S. (2014) 
Performance of SON for RSRP-Based LTE/WLAN Access Network Selection. Proc. Int. Symposium on Wireless Communications Systems, Barcelona, August 2014, 360-364.

[9] Irina, B., Daniela, L., Simone, R. and Andreas, L. (2015) RSRP-Based LTE-WLAN Traffic Steering. Proc. IEEE Vehcular Technology Conference, Glasgow, May 2015, 1-5.

[10] Wang, Y., Relja, D., Andreas, B., István, Z.K., Da- niela, L., Kathleen, S. and Bart, S. (2014) Performance of WLAN RSS-Based SON for LTE/WLAN Access Network Selection. Proc. Int. Symposium on Wireless Communications Systems, Barcelona, August 2014, 460-464. https://doi.org/10.1109/ISWCS.2014.6933397

[11] 3GPP TS 36.304 (2014) User Equipment (UE) Procedures in Idle Mode.

[12] 3GPP TR 36.814 (2010) Further Advancements for E-UTRA Physical Layer Aspects.

Submit or recommend next manuscript to SCIRP and we will provide best service for you:

Accepting pre-submission inquiries through Email, Facebook, LinkedIn, Twitter, etc. A wide selection of journals (inclusive of 9 subjects, more than 200 journals)

Providing 24-hour high-quality service

User-friendly online submission system

Fair and swift peer-review system

Efficient typesetting and proofreading procedure

Display of the result of downloads and visits, as well as the number of cited articles

Maximum dissemination of your research work

Submit your manuscript at: http://papersubmission.scirp.org/

Or contactijcns@scirp.org 